\title{
Differential expression and therapeutic efficacy of microRNA-346 in diabetic nephropathy mice
}

\author{
YONG ZHANG $^{1,2}$, HOU-QIN XIAO ${ }^{2}$, YANG WANG $^{3}$, ZHUO-SHUN YANG $^{4}$, \\ LONG-JUN DAI ${ }^{4,5}$ and YAN-CHENG XU ${ }^{1}$
}

\begin{abstract}
${ }^{1}$ Department of Endocrinology, Zhongnan Hospital of Wuhan University, Wuhan, Hubei 430071; ${ }^{2}$ Department of Nephrology, Taihe Hospital, Hubei University of Medicine, Shiyan, Hubei 442000; ${ }^{3}$ The Research Center for High Altitude Medicine, Medical College, Qinghai University, Xining, Qinghai 810001; ${ }^{4}$ Hubei Key Laboratory of Stem Cell Research, Taihe Hospital, Hubei University of Medicine, Shiyan, Hubei 442000, P.R. China; ${ }^{5}$ Department of Surgery, University of British Columbia, Vancouver, BC V5Z 1M9, Canada
\end{abstract}

Received August 7, 2014; Accepted April 27, 2015

DOI: $10.3892 / \mathrm{etm} .2015 .2468$

\begin{abstract}
Diabetic nephropathy (DN) is a major cause of end-stage renal disease, in which the SMAD signaling pathway plays an important role. The aim of the present study was to identify differentially expressed microRNAs (miRNAs) during the progression of DN and to investigate a selected miRNA in relation to SMAD3/4 and its therapeutic efficacy. The miRNA microarray was used to identify differentially expressed miRNAs in $d b / d b$ DN mice. Reverse transcription-quantitative polymerase chain reaction and immunoblot analyses were used to detect SMAD3/4 expression. The development of DN in the $d b / d b$ mice was demonstrated by glucose dysregulation and typical morphological changes in the kidney. miRNA-346 (miR-346) was identified as one of the differentially expressed miRNAs. The expression of SMAD3/4 was significantly attenuated by miR-346 administration and the therapeutic effects of miR-346 were observed in the DN mouse models. miR-346 was identified as a negative regulator of SMAD3/4. SMAD3/4 was upregulated in the renal tissue of $d b / d b$ mice. The administration of miR-346 attenuated the SMAD3/4 expression in renal tissue and ameliorated the renal function and glomerular histology in DN mice. This study paves the way for clinical studies of miR-346 in DN.
\end{abstract}

\section{Introduction}

Diabetes is the most prevalent disease worldwide. According to the World Health Organization, $\sim 9.5 \%$ of the adult population ( $\sim 347$ million individuals) were suffering from

Correspondence to: $\mathrm{Dr}$ Yan-Cheng $\mathrm{Xu}$, Department of Endocrinology, Zhongnan Hospital of Wuhan University, 169 Donghu Road, Wuhan, Hubei 430071, P.R. China

Email: oxyc@163.com

Key words: diabetes, microRNA microarray, diabetic nephropathy, microRNA-346 diabetes in 2008, and the number is expected to double by 2030 (1). As one of the major complications of diabetes, diabetic nephropathy (DN) is now the most common cause of end-stage renal disease, particularly in Western countries (2). Hypertension and poor glycemic control are the major clinical associations that frequently precede overt DN (3). In clinical terms, the condition is characterized by the development of proteinuria, specifically albuminuria, with a subsequent reduction in the glomerular filtration rate. DN can progress over a period of 10-20 years; if left untreated, the resulting uremia is fatal (4). The histological characteristics of DN include mesangial expansion, thickening of the glomerular basement membrane and podocyte effacement, in addition to glomerular sclerosis $(5,6)$. DN is one of the direct causes of mortality in diabetic patients. The $d b / d b$ mouse is an obese hyperinsulinemic model of genetic diabetes. This animal model develops abnormalities in renal function and renal morphology that parallel those in the nephropathy of patients with type 2 diabetes (7); therefore, the $d b / d b$ mouse is a useful animal model for investigating the pathophysiology of DN and exploring an effective therapy for the disease.

During the past two decades, a novel class of non-coding RNA, microRNAs (miRNAs), has been found to be expressed in all tissues and to play essential roles in tissue homeostasis and disease progression $(8,9)$. miRNAs are endogenous, single-stranded RNA molecules of $\sim 22$ nucleotides in length that regulate target mRNAs at the post-transcriptional level via base-pairing to target mRNAs. miRNAs are predicted to control the activity of $>60 \%$ of all protein-coding genes by repressing translation and/or bringing about the deadenylation and subsequent degradation of target mRNAs (10). Thus, miRNA deregulation often causes impaired cellular function and disease development. An enhanced understanding of the regulation and function of miRNAs in renal pathology could highlight certain miRNAs as novel therapeutic targets in kidney fibrosis and its associated diseases. In the present study, a high-throughput technology, the miRNA microarray, was utilized in order to identify DN-related miRNAs and determine the role of selected miRNAs in the progression of $\mathrm{DN}$, using $d b / d b$ DN mouse models. 


\section{Materials and methods}

Animals. C57BL/KsJ- $d b / d b$ mice and their littermate $d b / m$ mice were purchased from the Model Animal Research Center at Nanjing University (Nanjing, China) and housed in accordance with the National Institutes of Health Guide for the Care and Use of Laboratory Animals. The experimental protocols of the present study were approved by the Animal Care Committee at Hubei University of Medicine (Shiyan, China). The $d b / d b$ mice were determined to be diabetic on the basis of obesity and hyperglycemia at $\sim 4$ weeks of age. The mice were subject to right uninephrectomy under anesthesia at various time-points, depending on the experiment design. Anesthesia was performed using intraperitoneal injection of pentobarbital (90 mg/kg; Shanghai Chemical Reagent Co., Ltd., Shanghai, China), and the mice were sacrificed by cervical dislocation following anesthesia.

RNA isolation and miRNA microarray analysis. Mouse kidney tissue $(\sim 100 \mathrm{mg})$, taken from the renal cortices of DN or control mice, was added into $1 \mathrm{ml} \mathrm{TRIzol}{ }^{\circledR}$ reagent (Invitrogen Life Technologies, Carlsbad, CA, USA) in a 1.5-ml Eppendorf tube. The tissue was homogenized by RNase-free pestles followed by the addition of chloroform $(400 \mu \mathrm{l})$. Total RNA was extracted from the TRIzol-chloroform mixture using the miRNeasy Mini kit (Qiagen, Valencia, CA, USA). Total RNA was eluted in $100 \mu \mathrm{l}$ nuclease-free water, and the concentration was measured using the NanoDrop ${ }^{\mathrm{TM}} 8000$ spectrophotometer (Thermo Fisher Scientific, Inc., Wilmington, DE, USA). Samples with an $\mathrm{A}_{260} / \mathrm{A}_{280}$ ratio $>1.8$ were considered pure. The RNA integrity was verified by rRNA visualization following electrophoresis in an agarose gel.

miRNA microarray analysis. The differential expression profile of the miRNAs was assayed using the miRMouse miRNA array (Exiqon miRCURY LNA ${ }^{\mathrm{TM}}$ miRNA; Exiqon A/S, Vedbaek, Denmark), which contained 1,181 capture probes, in $\mathrm{db} / \mathrm{db}$ DN mice and $d b / m$ controls. Following RNA isolation from the samples, the microarray labeling and array hybridization procedure were performed according to the manufacturer's instructions. Upon the completion of the hybridization procedure, the slides were produced and dried by centrifugation. The slides were then scanned using a SureScan Microarray Scanner (Agilent Technologies, Santa Clara, CA, USA).

cDNA synthesis and quantitative polymerase chain reaction ( $q P C R)$ analysis. qPCR was performed to validate the miRNA expression profiles identified with the miRNA array. The first-strand cDNA was synthesized using the PrimeScript Reverse Transcription reagent kit (Clontech Laboratories, Inc., Mountain View, CA, USA) with oligonucleotide dTs. The cDNA levels for each tested miRNA and GAPDH gene transcripts were measured using a 7900HT Fast Real-Time PCR system (Applied Biosystems, Foster City, CA, USA) according to the manufacturer's instructions. Each amplification reaction was performed in triplicate in separate tubes. The average calculated from the three threshold cycles was used to determine the number of transcripts in the sample. miRNA expression was quantitatively expressed in arbitrary units as the ratio of the target sample quantity to the calibrator or to the mean values of the control samples. All values were normalized to the endogenous control GAPDH. qPCR was also used to detect SMAD3/4 expression, with primers provided by Sangon Biotech Co., Ltd. (Shanghai, China).

miR-346-bearing plasmid construction and administration. The miR-346 expression vector was constructed according to the miR-346 sequence (accession no. MI0000634; http://www.mirbase.org/) using the SacI cloning site of pGenesil-1. Recombinant pGenesil-miR-346 and control pGenesil-1 were provided by Guangzhou RiboBio Co., Ltd. (Guangzhou, China). Starting at week 8, three groups of $d b / d b$ DN mice (6 mice/group) were intravenously injected an miR-346-bearing plasmid (pGenesil-miR-346, $30 \mathrm{mg} / \mathrm{kg}$ ), a plasmid control (pGenesil-1, $30 \mathrm{mg} / \mathrm{kg}$ ) and an untreated control (same volume of saline), respectively. The injection was administered through the tail vein once a week until week 16 . The urinary protein content, body weight and blood glucose were dynamically monitored. At week 16, all animals were sacrificed, and their renal cortex tissues were sampled for molecular analysis and morphological examinations.

Immunoblot analysis. Immunoblot analysis was used to detect SMAD3/4 expression in the renal tissues. The renal tissue samples were harvested from miR-346-treated (pGenesil-miR-346), plasmid-treated (pGenesil-1) and untreated control mice at week 16 . The tissue samples were homogenized with Kontes pellet pestle cordless motor (Thermo Fisher Scientific, Inc.) and lysed with cell lysis solution (Bio-Rad Laboratories, Inc., Hercules, CA, USA), and the tissue lysates were subjected to an immunoblotting analysis as previously described (11). The total protein was quantified using Pierce BCA protein assay kit (Life Technologies). Briefly, tissue lysates $(50 \mu \mathrm{g})$ were separated through $12 \%$ denaturing sodium dodecyl sulfate-polyacrylamide gel electrophoresis and transferred to a nitrocellulose membrane. The membrane was incubated overnight with rabbit anti-SMAD3/4 polyclonal antibodies (1:1,000; \#9523; Cell Signaling Technology, Inc., Boston, MA, USA). After three washes with Tris-buffered saline $/ 0.05 \%$ Tween-20, the membrane was incubated for $1 \mathrm{~h}$ with goat anti-rabbit immunoglobulin $\mathrm{G}$ conjugated to horseradish peroxidase (1:2,500; Santa Cruz Biotechnology, Inc., Santa Cruz, CA, USA). Then, the membrane was washed three times with Tris-buffered saline $/ 0.05 \%$ Tween-20, followed by a final wash with Tris-buffered saline without Tween-20. The blot was developed using enhanced chemiluminescence detection (Beyotime Institute of Biotechnology, Shanghai, China). $\beta$-actin was used as a loading control.

Renal tissue staining. The Periodic acid Schiff (PAS) protocol was used for renal tissue staining. Tissue sections $(\sim 5 \mu \mathrm{m})$ were mounted on slides and stained with a PAS staining kit (Sigma-Aldrich, St. Louis, MO, USA), as per the manufacturer's instructions.

Statistical analysis. Scanned images from the microarray were imported into miRCURY LNA software (Exiqon A/S) for grid alignment and data extraction. Expressed data were normalized using median normalization. The numerical results are expressed as the mean \pm standard error of the mean, with ' $n$ ' 
representing the number of animals. Groups were compared using one-way analysis of variance and subsequently using the Student-Newman-Keuls and Dunnett tests for multiple comparisons. $\mathrm{P}<0.05$ was considered to indicate a statistically significant difference.

\section{Results}

Demonstration of DN in $\mathrm{db} / \mathrm{db}$ mice. The blood glucose, body weight and urinary proteins of the DN mice were dynamically observed for 16 weeks (Table I). Starting from week 4, the blood glucose level and body weight of the $d b / d b$ mice were significantly higher than those of the $d b / m$ control mice $(\mathrm{P}<0.05)$, thereby confirming the development of $\mathrm{DN}$ in the $d b / d b$ mice. Fig. 1 shows the glomerular pathologies of the $d b / d b$ and $d b / m$ mice at the end of the observation period (week 18). Typical DN changes were observed in the $d b / d b$ mouse kidney, including glomerular hypertrophy, mesangial cell proliferation and matrix accumulation. The results indicated the development of DN in the $d b / d b$ mice.

miRNA profiling of the $d b / d b$ mice. An miRNA microarray was used to identify the miRNA profiles of the mouse DN model. To ensure the reliable performance of the microarray and qPCR, RNA samples isolated from the renal cortices of DN and control animals were verified prior to the miRNA array being performed. The RNA purity was confirmed by a $260 / 280$ optical density value of $1.8-2.0$ in both DN $(n=6)$ and control groups $(n=6)$. The RNA samples were also assessed for degradation status by agarose gel electrophoresis. Three RNA samples were randomly selected from the DN and control groups and were subjected to subsequent $\mathrm{Cy} 3$ and $\mathrm{Cy} 5$ labeling and hybridization with the Exiqon miRNA array. The microarray data demonstrated that the $d b / d b$ DN mice had a significantly altered miRNA profile compared with the $\mathrm{db} / \mathrm{m}$ control mice. An overview of the miRNA expression is shown in Fig. 2. In total, 772 and 433 miRNAs were detected for expression in the DN and control mice, respectively. Compared with the control, 219 miRNAs were differentially expressed by $>2$-fold, and 71 miRNAs had a difference of >4-fold in the DN mice. Among these 71 miRNAs, 39 miRNAs were upregulated (Fig. 3A) and 31 miRNAs were downregulated (Fig. 3B).

To validate the expression profile obtained from the miRNA microarray analysis, 10 downregulated miRNAs (miR-215, miR-451, miR-200a, miR-200b, miR-200c, miR-141, miR-21, miR-346, miR-709 and miR-187) and 8 upregulated miRNAs (miR-29c, miR-192, miR-216a, miR-17, miR-377, miR-196a, miR-166 and miR-98) were quantitatively examined using qPCR analysis. As shown in Table II, consistent fold changes were observed between the qPCR and microarray for the selected miRNAs, indicating the reliability of the miRNA microarray analysis under the present experimental conditions.

miR-346 target prediction. Target predictions were based on the detected expression profiles of the miRNAs. Considering glomerular fibrosis as a major process during the pathogenesis of DN, the target searching was focused on fibrosis-related mRNAs. As guided by Krek et al (12), the PicTar algorithm and the microRNA.org website (http://www.microrna.org/) were used to identify potential mRNA targets of the miRNAs found to
Table I. Dynamic observation of body weight, blood glucose and proteinuria.

\begin{tabular}{lccc}
\hline Time-point & Body weight $(\mathrm{g})$ & $\begin{array}{c}\text { Blood glucose } \\
(\mathrm{mmol} / \mathrm{l})\end{array}$ & $\begin{array}{c}\text { Proteinuria } \\
(\mu \mathrm{g} / 24 \mathrm{~h})\end{array}$ \\
\hline Week 4 & & & \\
Control & $15.11 \pm 1.64$ & $6.45 \pm 0.75$ & $8.76 \pm 2.01$ \\
DN & $18.65 \pm 1.23^{\mathrm{a}}$ & $8.75 \pm 1.45^{\mathrm{a}}$ & $9.54 \pm 2.43$ \\
Week 8 & & & \\
Control & $18.23 \pm 1.46$ & $6.92 \pm 0.56$ & $9.65 \pm 2.38$ \\
DN & $37.38 \pm 1.94^{\mathrm{a}}$ & $26.45 \pm 3.12^{\mathrm{a}}$ & $89.13 \pm 12.64^{\mathrm{a}}$ \\
Week 12 & & & \\
Control & $21.71 \pm 1.92$ & $7.75 \pm 1.46$ & $10.01 \pm 2.48$ \\
DN & $46.82 \pm 2.32^{\mathrm{a}}$ & $36.42 \pm 4.12^{\mathrm{a}}$ & $158.73 \pm 22.16^{\mathrm{a}}$ \\
Week 16 & & & \\
Control & $24.56 \pm 1.86$ & $8.21 \pm 1.66$ & $10.54 \pm 3.14$ \\
DN & $50.12 \pm 2.75^{\mathrm{a}}$ & $40.98 \pm 4.77^{\mathrm{a}}$ & $209.72 \pm 22.88^{\mathrm{a}}$ \\
\end{tabular}

*Compared with the control, $\mathrm{P}<0.05$. DN, diabetic nephropathy.

Table II. Fold changes in the miRNA expression in DN mice, as determined by qPCR and microarray analyses.

\begin{tabular}{lrr}
\hline miRNAs & qPCR $^{\mathrm{a}}$ & miRNA array $^{\mathrm{b}}$ \\
\hline Downregulated miRNAs & & \\
miRNA-215 & -4.23 & -5.11 \\
miRNA-346 & -8.12 & -8.20 \\
miRNA-200a & -12.32 & -13.76 \\
miRNA-200b & -14.13 & -10.22 \\
miRNA-200c & -13.11 & -7.75 \\
miRNA-141 & -6.14 & -8.13 \\
miRNA-21 & -2.55 & -4.76 \\
miRNA-45 1 & -1.89 & -4.12 \\
miRNA-709 & -2.33 & -5.54 \\
miRNA-187 & -7.32 & -6.93 \\
Upregulated miRNAs & & \\
miRNA-29c & 3.45 & 5.67 \\
miRNA-192 & 2.11 & 4.35 \\
miRNA-216a & 1.98 & 5.76 \\
miRNA-166 & 10.12 & 8.20 \\
miRNA-17 & 5.44 & 6.45 \\
miRNA-377 & 7.88 & 10.12 \\
miRNA-196a & 4.65 & 4.32 \\
miRNA-98 & 2.35 & 4.03 \\
\hline
\end{tabular}

${ }^{\mathrm{a}} \mathrm{qPCR}$ results were calculated using the $2^{-\Delta \triangle \mathrm{CT}}$ method; ${ }^{\mathrm{b}}$ microarray results represent the ratios of the DN/control. miRNA, microRNA; DN, diabetic nephropathy; qPCR, quantitative polymerase chain reaction.

be differentially regulated in the DN mice. Among the 71 most differentially expressed miRNAs, miR-346 was identified a SMAD family-related miRNA that has not been well characterized in this regard. Of the 95 potential targets for miR-346, SMAD3 and SMAD4 were closely associated with fibrogenesis. As shown in Fig. 4, miR-346 has 6-11 complementary binding sites in the 3'-untranslated region (3'-UTR) of SMAD3/4. 
A

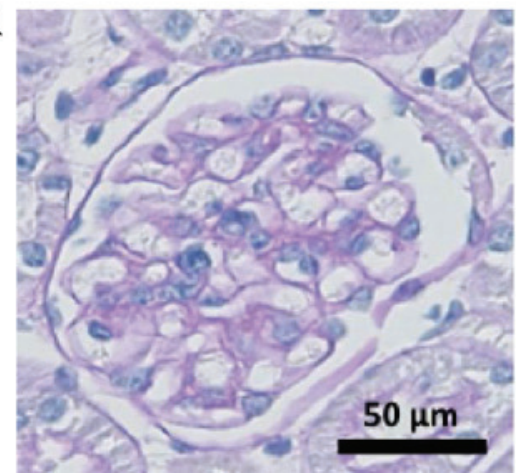

B

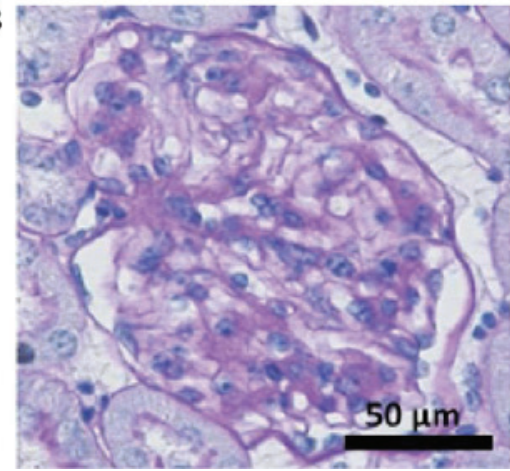

Figure 1. Pathological glomerular changes. (A) $d b / m$ and (B) $d b / d b$ mice were sacrificed at week 18 , and the renal tissue sections were stained with Periodic acid Schiff.

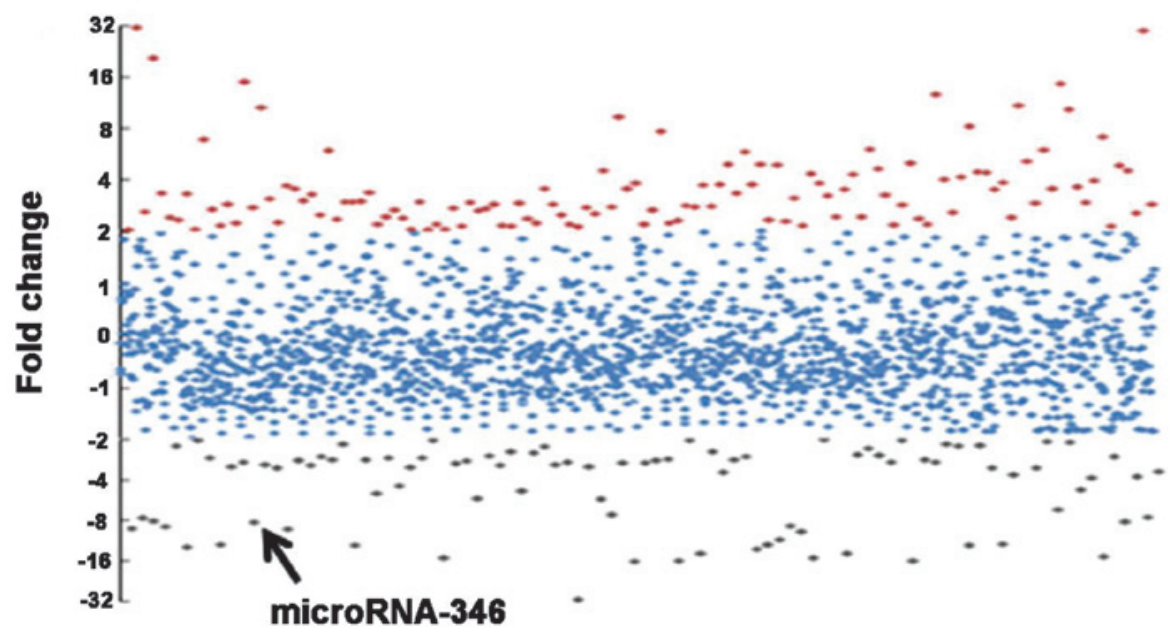

Figure 2. Overview of miRNA expression in the renal tissue of DN mice. Red spots represent miRNAs upregulated $>2$-fold; grey spots represent miRNAs downregulated $>2$-fold; and blue spots represent miRNAs that are less differentially expressed, with a fold change of between +2 and -2 . The fold change of miR-346 is indicated. miRNA, microRNA; miR-346, microRNA-346; DN, diabetic nephropathy.

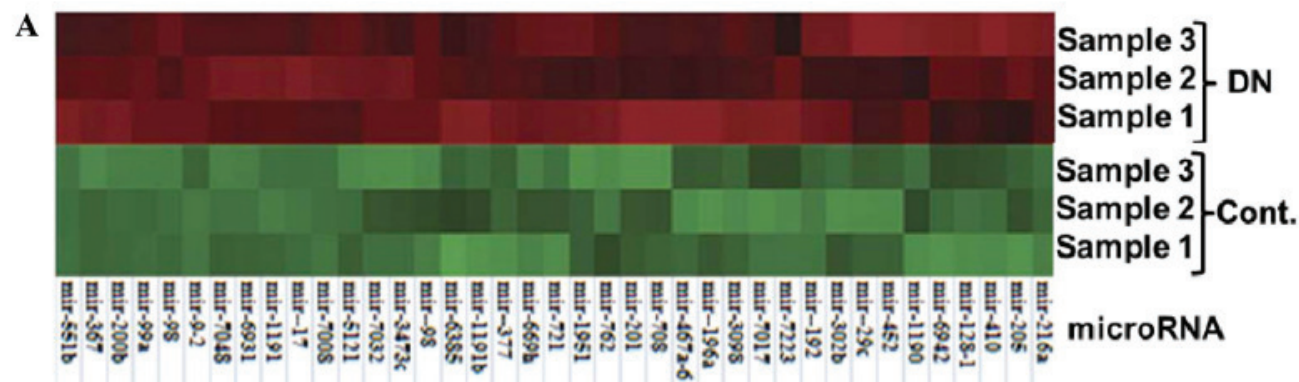

B

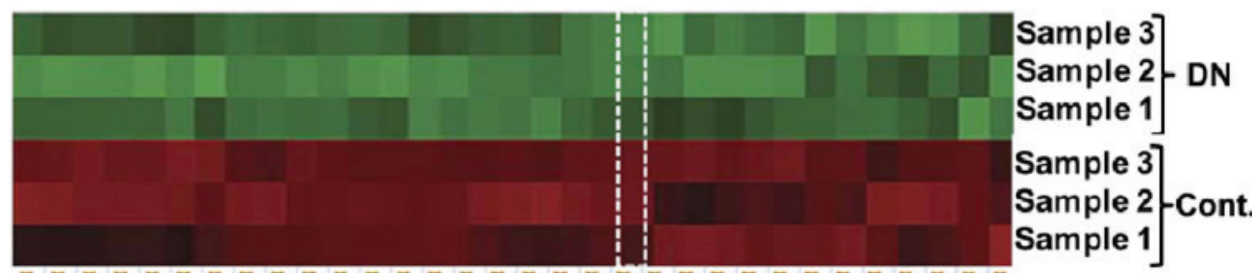

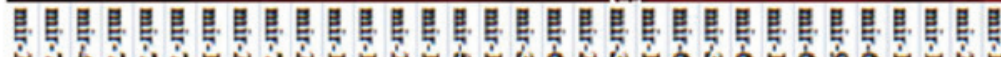

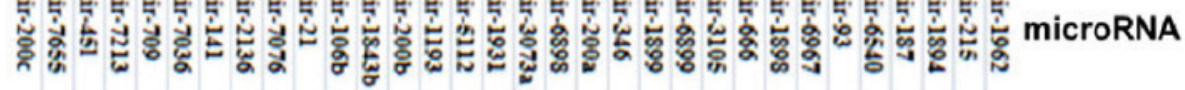

Figure 3. Differentially expressed miRNAs in the renal tissue of DN mice. Isolated miRNAs from the renal tissues of $d b / m$ control and $d b / d b$ DN mice were labeled with Cy3 (green) and Cy5 (red), respectively, prior to hybridization with a microRNA array. The color change was determined by the ratio of Cy3/Cy5. (A) Thirty-nine upregulated miRNAs; (B) 32 downregulated miRNAs (miR-346 is highlighted). The array information was obtained from Sanger miRBase Release 18.0 (http://www.mirbase.org). miRNA, microRNA; miR-346, microRNA-346; DN, diabetic nephropathy; Cont., control. 


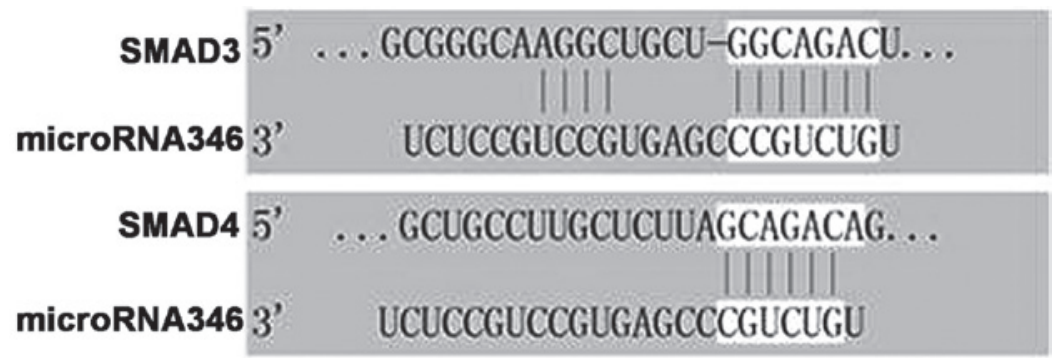

Figure 4. Complementary binding sites between miR-346 and SMAD3/4.

A

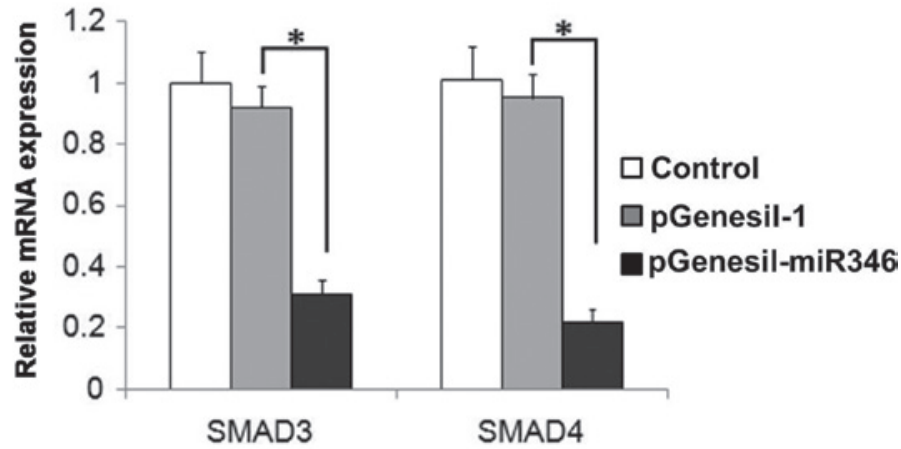

B

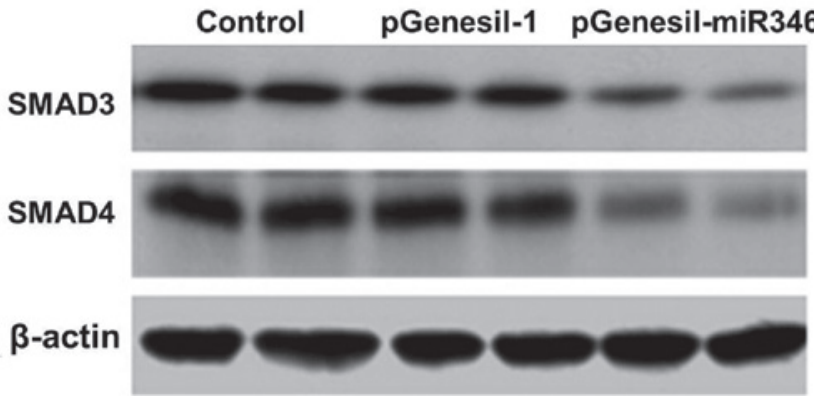

Figure 5. Demonstration of SMAD3/4 expression in renal tissues. (A) mRNA expression determined through reverse transcription-quantitative polymerase chain reaction analysis; (B) protein expression assessed by immunoblotting analysis. ${ }^{*} \mathrm{P}<0.05$.

Effect of miR-346 on SMAD3/4 expression in the renal tissue of DN mice. As shown in Fig. 5A and B, the expression of SMAD3/4 was significantly attenuated by miR-346 administration, which was demonstrated in the analysis of both mRNA and protein levels.

Therapeutic efficacy of miR-346 in db/db DN mice. As shown in Fig. 6A, a significant improvement in the proteinuria was observed with pGenesil-miR-346 administration, although the changes in the body weight and blood glucose level remained consistent with the controls. As can be noted in Fig. 6B, typical DN glomerular alterations were present in both the plasmid-treated and untreated controls, exhibiting matrix accumulation, glomerular hypertrophy and mesangial cell proliferation; however, the $d b / d b$ mice treated with pGenesil-miR-346 exhibited a normal glomerular histology.

\section{Discussion}

DN as a consequence of types 1 and 2 diabetes is the major cause of end-stage renal disease worldwide. The glomerular histology is characterized by mesangial expansion, glomerular basement membrane thickening and glomerular sclerosis (13). Mesangial fibrogenesis plays an important role during DN progression, and the SMAD signaling pathway is widely recognized to be a major regulator responsible for the fibrogenesis. Several miRNAs have been proven to be closely associated with DN, and their pathophysiological effects in chronic kidney disease have been studied, including miR-192 (14), miR-29a (15), miR-377 (16), miR-200a, miR-200b, miR-205, miR-141 and miR-429 (17). The C57BL/KsJ- $d b / d b(d b / d b)$ mouse is one of the most widely used models for diabetes-induced nephropathy research. In the present study, the expression of 772 miRNAs was detected in the renal tissues of $d b / d b$ DN mice, and 219 of the miRNAs were differentially expressed $>2$-fold compared with the $d b / m$ control mice (Fig. 2). The miRNA microarray results were verified by confirmatory qPCR (Table II). Among the 71 most differentially expressed miRNAs, miR-346 was identified as a SMAD family-related miRNA that has not been well characterized. Through target prediction analysis, SMAD3 and SMAD4 (SMAD3/4) were identified to be in the list of 95 potential targets for miR-346. 

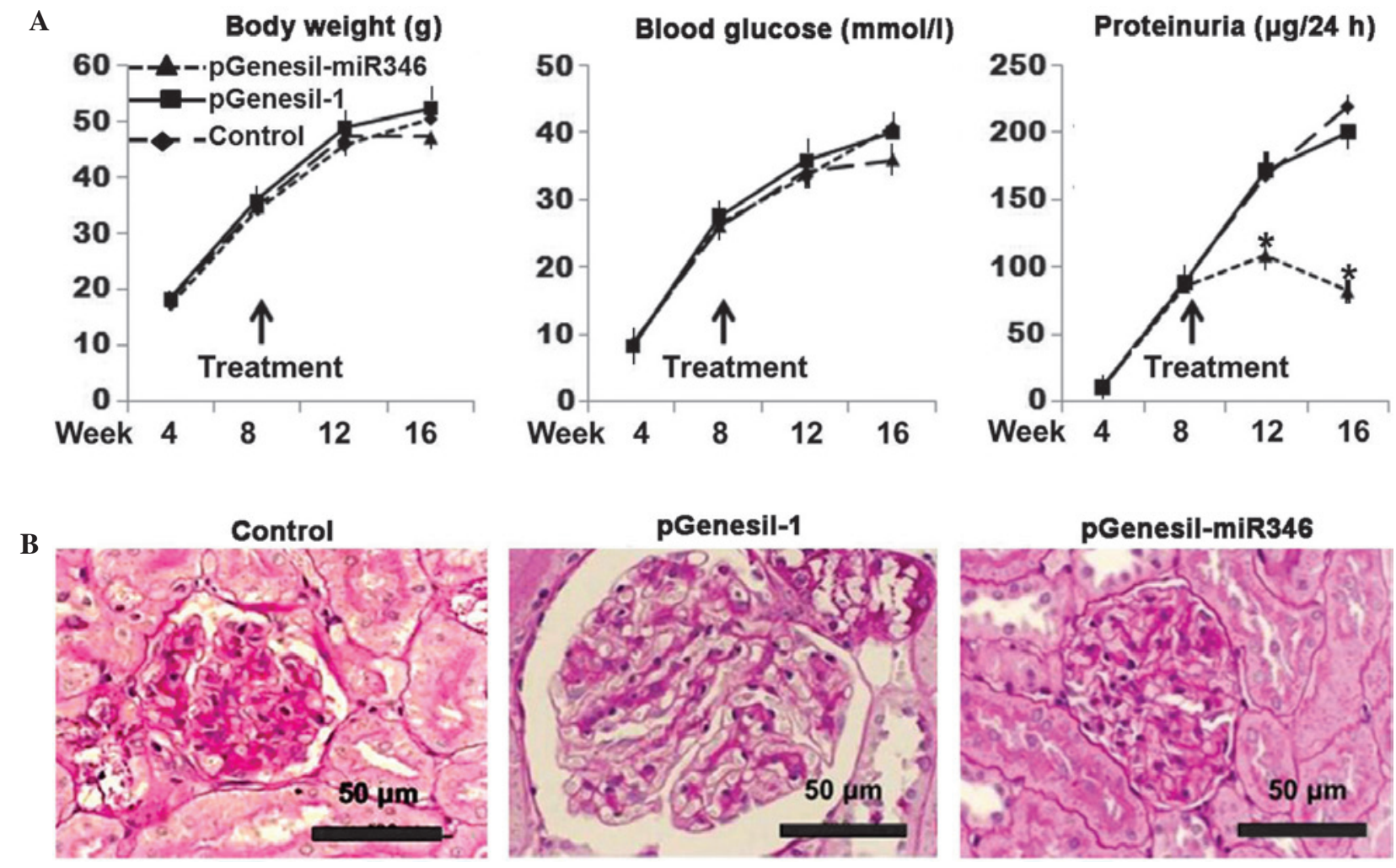

Figure 6. Therapeutic effects of miR-346 on mouse diabetic nephropathy. (A) miR-346 treatment induced physiological changes in $d b / d b$ mice. The treatment was initiated at week 8 . Each data-point represents the mean value of 3 observations ( $n=3)$. " $\mathrm{P}<0.05$ vs. control. (B) Protective effect of miR-346 on the glomerular histology in $d b / d b$ mice. $d b / d b$ mice were sacrificed at week 16 , and renal tissue sections were stained with Periodic acid Schiff. miR-346, microRNA-346.

There existed 6-11 complementary binding sites in the 3'-UTR of SMAD3/4 (Fig. 4). To the best of our knowledge, this is the first time that miR346 has been identified as a regulator of SMAD3/4.

The regulatory action of miRNA is mainly achieved through the competitive crosstalk between miRNA and the targeted mRNA (18). The primary underlying mechanism involves the interaction between the miRNAs and the 3'-UTR of protein-coding genes; this interaction typically results in a decrease in protein output, either by mRNA degradation or by translational repression, depending on the extent of the complementary binding (19). As demonstrated in Fig. 5, the miR-346-induced inhibition of SMAD3/4 exhibited the same pattern in both mRNA and protein levels, suggesting that miR-346-induced SMAD3/4 mRNA degradation could be involved in the action. SMAD3/4 plays an important role in transforming growth factor (TGF)- $\beta$-mediated fibrogenesis through SMAD3/4 interactions with the promoter of the collagen type $1 \alpha 2$ gene $(20,21)$. TGF- $\beta$ has been considered to be a key mediator in renal fibrosis, predominantly through the activation of its downstream SMAD signaling pathway. The emerging role of miRNAs in TGF- $\beta$-mediated renal fibrosis has been documented in the past 5 years (22). TGF- $\beta 1$-upregulated miRNAs include miR-377, miR-21, miR-93, miR-216a and miR-192, whereas the miR-29 and miR-200 families are downregulated by TGF- $\beta 1$. Notably, the majority of this research has focused on miRNAs that target molecules involved in renal fibrosis via effects elicited by TGF- $\beta$ (4); however, the interac- tion between miR-346 and TGF- $\beta$ has not been explored in the present study.

In this study, an miR-346-bearing plasmid was used to verify the therapeutic efficacy of miR-346 on DN in $d b / d b$ mice. During the period of treatment (up to 8 weeks) the blood glucose level and body weight were not affected by the administration of miR346 (Fig. 6A); however, almost from the initiation of the treatment, the proteinuria was significantly improved. At the end of the experiment, the glomerular histology was almost completely restored to normal (Fig. 6B). The results confirmed the therapeutic efficacy of miR-346 on DN in this particular animal model. Further mechanism-related studies may lead to the development of effective therapies for clinical DN.

In conclusion, miR-346 was identified for the first time, to the best of our knowledge, as a negative regulator of SMAD3/4. SMAD3/4 was upregulated in the renal tissue of $d b / d b$ mice. The administration of miR-346 attenuated the SMAD3/4 expression in the renal tissue and ameliorated the renal function and glomerular histology in the DN mice. The identification and therapeutic efficacy of miR-346 in a $d b / d b$ mouse model may pave the way for clinical studies of miR-346 in DN.

\section{Acknowledgements}

This study was supported by Taihe Hospital Foundation. The authors are grateful to James Dai (LJ Resources Co., Ltd., 
Vancouver, Canada) for his assistance in preparing the manuscript and English proofreading.

\section{References}

1. Danaei G, Finucane MM,Lu Y, et al; Global Burden of Metabolic Risk Factors of Chronic Diseases Collaborating Group (Blood Glucose): National, regional, and global trends in fasting plasma glucose and diabetes prevalence since 1980: Systematic analysis of health examination surveys and epidemiological studies with 370 country-years and 2.7 million participants. Lancet 378: 31-40, 2011.

2. Gilbertson DT, Liu J, Xue JL, et al: Projecting the number of patients with end-stage renal disease in the United States to the year 2015. J Am Soc Nephrol 16: 3736-3741, 2005.

3. Hagiwara S, McClelland A and Kantharidis P: MicroRNA in diabetic nephropathy: Renin angiotensin, aGE/RAGE, and oxidative stress pathway. J Diabetes Res 2013: 173783, 2013.

4. Forbes JM and Cooper ME: Mechanisms of diabetic complications. Physiol Rev 93: 137-188, 2013.

5. Adler S: Diabetic nephropathy: Linking histology, cell biology, and genetics. Kidney Int 66: 2095-2106, 2004.

6. Susztak K, Raff AC, Schiffer M and Böttinger EP: Glucose-induced reactive oxygen species cause apoptosis of podocytes and podocyte depletion at the onset of diabetic nephropathy. Diabetes 55: 225-233, 2006.

7. Sharma K, McCue P and Dunn SR: Diabetic kidney disease in the $\mathrm{db} / \mathrm{db}$ mouse. Am J Physiol Renal Physiol 284: F1138-F1144, 2003

8. Yates LA, Norbury CJ and Gilbert RJ: The long and short of microRNA. Cell 153: 516-519, 2013.

9. Soifer HS, Rossi JJ and Saetrom P: MicroRNAs in disease and potential therapeutic applications. Mol Ther 15: 2070-2079, 2007.

10. Fabian MR, Sonenberg N and Filipowicz W: Regulation of mRNA translation and stability by microRNAs. Annu Rev Biochem 79: 351-379, 2010.
11. Yang ZS, Tang XJ, Guo XR, et al: Cancer cell-oriented migration of mesenchymal stem cells engineered with an anticancer gene (PTEN): An imaging demonstration. Onco Targets Ther 7: 441-446, 2014.

12. Krek A, Grün D, Poy MN, et al: Combinatorial microRNA target predictions. Nat Genet 37: 495-500, 2005.

13. Lorenzen JM, Haller H and Thum T: MicroRNAs as mediators and therapeutic targets in chronic kidney disease. Nat Rev Nephrol 7: 286-294, 2011.

14. Krupa A, Jenkins R, Luo DD, et al: Loss of MicroRNA-192 promotes fibrogenesis in diabetic nephropathy. J Am Soc Nephrol 21: 438-447, 2010.

15. Du B, Ma LM, Huang MB, Zhou H, Huang HL, Shao P, Chen YQ and Qu LH: High glucose down-regulates miR-29a to increase collagen IV production in HK-2 cells. FEBS Lett 584: 811-816, 2010.

16. Wang Q, Wang Y, Minto AW, et al: MicroRNA-377 is up-regulated and can lead to increased fibronectin production in diabetic nephropathy. FASEB J 22: 4126-4135, 2008.

17. Wang G, Kwan BC, Lai FM, et al: Intrarenal expression of miRNAs in patients with hypertensive nephrosclerosis. Am J Hypertens 23: 78-84, 2010.

18. Tay Y, Rinn J and Pandolfi PP: The multilayered complexity of ceRNA crosstalk and competition. Nature 505: 344-352, 2014.

19. Ling H, Fabbri $M$ and Calin GA: MicroRNAs and other non-coding RNAs as targets for anticancer drug development. Nat Rev Drug Discov 12: 847-865, 2013.

20. Meng XM, Huang XR, Chung AC, et al: Smad2 protects against TGF-beta/Smad3-mediated renal fibrosis. J Am Soc Nephrol 21: 1477-1487, 2010.

21. Meng XM, Chung AC and Lan HY: Role of the TGF- $\beta$ /BMP-7/Smad pathways in renal diseases. Clin Sci (Lond) 124: 243-254, 2013.

22. Kantharidis P, Wang B, Carew RM and Lan HY: Diabetes complications: The microRNA perspective. Diabetes 60: 1832-1837, 2011. 